\title{
The Sagnac Effect in curved space-times from an analogy with the Aharonov-Bohm Effect
}

\author{
Matteo Luca Ruggiero ${ }^{1,2}$ \\ ${ }^{1}$ Dipartimento di Fisica, Politecnico di Torino, \\ ${ }^{2}$ INFN, Sezione di Torino \\ e-mail: matteo.ruggiero@polito.it
}

August 27, 2018

\begin{abstract}
In the context of the natural splitting, the standard relative dynamics can be expressed in terms of gravito-electromagnetic fields, which allow to formally introduce a gravito-magnetic Aharonov-Bohm effect. We showed elsewhere that this formal analogy can be used to derive the Sagnac effect in flat space-time as a gravito-magnetic AharonovBohm effect. Here, we generalize those results to study the General Relativistic corrections to the Sagnac effect in some stationary and axially symmetric geometries, such as the space-time around a weakly gravitating and rotating source, Kerr space-time, Gödel universe and Schwarzschild space-time.
\end{abstract}

Keywords: Sagnac Effect, Gravitoelectromagnetism, Aharonov-Bohm Effect, GravitoMagnetic Aharonov-Bohm Effect.

PACS: 04.20.-q.

\section{Introduction}

In previous papers [1, 2 we showed that the standard relative dynamics, in the background of the natural splitting developed by Cattaneo [3, 4, 5, 6, 7, can be described in terms of a gravito-electromagnetic (GEM) formal analogy. In other words, the time+space splitting allows to write the equation of motion of a particle, in arbitrary space-times, in analogy with the equation of motion of a charged particle acted upon by a "generalized" Lorentz force, in flat space-time. 
This analogy holds in full theory and, in linear approximation, it corresponds to the well known analogy between the theory of electromagnetism and the linearized theory of General Relativity (GR) [8]. Indeed, gravitoelectromagnetism in relativistic gravity is somewhat ubiquitous, because of its "many faces" [9, and various approaches prove useful for studying gravitational phenomena in terms of electromagnetic ones [10, 11].

Our GEM approach to the study of relativistic dynamics leads to what we called gravito-magnetic (GM) Aharonov-Bohm effect. Indeed, in the past other authors [12, 13, 14, 15, 16, 17, 18, suggested the possibility of describing the phase shift and time delay, induced by inertial and gravitational fields, in terms of an analogy with the Aharonov-Bohm effect for the electromagnetic field [19]. Our formalism applies to both inertial and gravitational fields, holds in full theory and generalizes the previous results, which were obtained, almost always, at first order approximation (with respect to $c$, i.e. in the slow motion approximation).

We already used this approach to obtain a derivation "by analogy" of the Sagnac phase shift and time delay, for matter or light beams counterpropagating on a round trip, in a rotating reference frame in flat space-time 1, 2, and our results are in agreement with the fully relativistic treatment of the Sagnac effect given by Anandan [20. In doing so, we exploited the formal analogy between matter beams (or light beams) counter propagating along circular trajectories in inertial fields, and charged beams propagating in a region where a magnetic vector potential is present. In other words, we showed that the Sagnac effect may be thought of as a GM Aharonov-Bohm effect.

Here we are concerned with the generalization of those results to curved space-times: we study the Sagnac effect in the space-time around a weakly gravitating and rotating source, in Kerr space-time, in Gödel universe and Schwarzschild space-time, and we derive the Sagnac time-delay by following the analogy with the Aharonov-Bohm effect. ${ }^{1}$ In doing so, we recover some old results 23, 24, 25, and suggest that our approach can be applied to arbitrary stationary and axially symmetric geometries. 


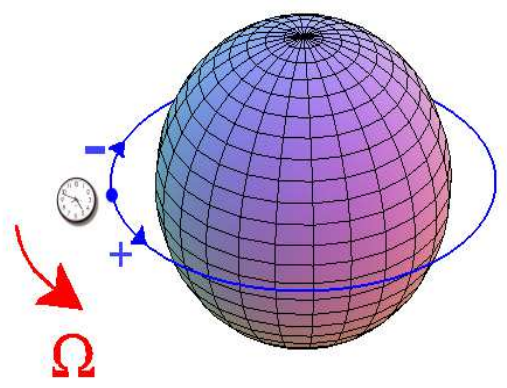

Figure 1: We consider the proper time difference (as measured by a clock rotating with constant angular velocity $\Omega$ ) between the emission and absorption of the copropagating $(+)$ and counter-propagating (-) beam.

\section{Sagnac effect in curved space-times}

The Sagnac effect has been thoroughly studied in the past, and it has been detected in many experiments (see [2] for a recent review). It is well known that when observing the interference between light or matter beams (such as light beams, electron or neutron beams and so on) counter-propagating in flat space-time along a closed path in a rotating interferometer a fringe shift $^{2} \Delta \Phi$ arises. This phase shift can be interpreted as a time difference between the propagation times (as measured by a clock at rest on the rotating interferometer) of the co-rotating and counter-rotating beam.

By applying Cattaneo's splitting, the dynamics of massive or massless particles, relative to a given time-like congruence $\Gamma$ of unit vectors $\gamma(x)$, can be described in terms of a "gravito-electromagnetic" fields [2]. By exploiting this fact, in [1] we already showed that the Sagnac effect, for both matter and light beams, counter-propagating on a round trip in a interferometer rotating in flat space-time, may be obtained by following a formal analogy with the Aharonov-Bohm effect. This procedure can be generalized to study the Sagnac effect in curved space-time, in order to obtain the GR corrections. Then, we can study the interference process of matter or light beams in a

\footnotetext{
${ }^{1}$ Several descriptions of the Aharonov-Bohm effect have been developed (see for instance [21); as for the Sagnac time-delay, we follow and extend the approach already considered in 22].

${ }^{2}$ With respect to the interference pattern when the device is at rest.
} 
rotating frame in curved space-time in terms of the GM Aharonov-Bohm effect. In other words, this corresponds to calculating the GM AharonovBohm phase shift (see [1, 2])

$$
\Delta \Phi=\frac{2 m \gamma_{0}}{c \hbar} \oint_{C} \widetilde{\boldsymbol{A}}_{G} \cdot \mathrm{d} \widetilde{\boldsymbol{x}}=\frac{2 m \gamma_{0}}{c \hbar} \int_{S} \widetilde{\boldsymbol{B}}_{G} \cdot \mathrm{d} \widetilde{\boldsymbol{S}}
$$

detected by a uniformly rotating interferometer, and the time difference

$$
\Delta T=\frac{2 \gamma_{0}}{c^{3}} \oint_{C} \widetilde{\boldsymbol{A}}_{G} \cdot \mathrm{d} \widetilde{\boldsymbol{x}}=\frac{2 \gamma_{0}}{c^{3}} \int_{S} \widetilde{\boldsymbol{B}}_{G} \cdot \mathrm{d} \widetilde{\boldsymbol{S}},
$$

measured by a comoving observer provided with a standard clock.

The time difference (2) corresponds to the Sagnac time-delay: we notice that its expression clearly evidences the "universal" character of the Sagnac effect, since the mass $m$, or more correctly, the energy ${ }^{3}$ of the particles of the interfering beams does not appear. So, the Sagnac effect turns out to be an effect of the geometry of space-time, and it can be considered universal, in the sense that it is the same, independently of the physical nature of the interfering beams.

As we said, eq. (2) expresses the delay, for a round trip, between the propagation time of the co-propagating and counter-propagating beam (see Figure 11). In what follows, the two beams are supposed to have the same velocity (in absolute value) with respect to the rotating frame. Furthermore, the particles of the beams are supposed to be spinless, or their spins are neglected. ${ }^{4}$

It is easy to recognize that this analogy between the Sagnac effect and the Aharonov-Bohm effect holds in arbitrary stationary and axially symmetric geometries. Hence, in these cases, for studying the Sagnac effect it is sufficient to express the space-time metric in coordinates adapted to a congruence of rotating observers. Generally speaking, the metrics we deal with are given in coordinates adapted to a congruence of asymptotically inertial observers; if the coordinates are spherical, the passage to a congruence of observers uniformly rotating in the equatorial plane is obtained by applying

\footnotetext{
${ }^{3}$ In (11) $m$ is the standard relative mass or standard relative energy of the particles of the beams (see [2]).

${ }^{4}$ Indeed, things are different when a particle with spin, moving in a rotating frame or around a rotating mass, is considered. In this case a coupling between the spin and the angular velocity of the frame or the angular momentum of the rotating mass appears (this effect is evaluated by Hehl-Ni [26, Mashhoon 27] and Papini [28]). Hence, the formal analogy leading to the formulation of the GM Aharonov-Bohm effect holds only when the spin-rotation coupling is neglected.
} 
the (azimuthal) coordinates transformation

$$
\left\{\begin{aligned}
c t & =c t^{\prime} \\
r & =r^{\prime} \\
\varphi & =\varphi^{\prime}-\Omega t^{\prime} \\
\vartheta & =\vartheta^{\prime}
\end{aligned}\right.
$$

where $\Omega$ is the (constant) angular velocity.

Then, it is simple to apply the formalism that we have already used in 1, 2]: the following steps give a prescription for calculating the Sagnac effect in arbitrary stationary and axially symmetric geometries in both flat and curved space-times. ${ }^{5}$

- Define the time-like congruence $\Gamma$ of rotating observers.

- Express the space-time metric in coordinates adapted to congruence of rotating observers.

- Calculate the unit vectors field $\gamma(x)$

$$
\left\{\begin{array} { l } 
{ \gamma ^ { 0 } \doteq \frac { 1 } { \sqrt { - g _ { 0 0 } } } , } \\
{ \gamma ^ { i } \doteq 0 , }
\end{array} \quad \left\{\begin{array}{l}
\gamma_{0} \doteq-\sqrt{-g_{00}} \\
\gamma_{i} \doteq g_{i 0} \gamma^{0}
\end{array}\right.\right.
$$

- Calculate the gravito-magnetic vector potential

$$
\widetilde{A}_{i}^{G} \doteq c^{2} \frac{\gamma_{i}}{\gamma_{0}}
$$

- Calculate the Sagnac time-delay as a GM Aharonov-Bohm effect

$$
\Delta T=\frac{2 \gamma_{0}}{c^{3}} \oint_{C} \widetilde{\boldsymbol{A}}_{G} \cdot \mathrm{d} \widetilde{\boldsymbol{x}}=\frac{2 \gamma_{0}}{c^{3}} \int_{S} \widetilde{\boldsymbol{B}}_{G} \cdot \mathrm{d} \widetilde{\boldsymbol{S}}
$$

We want to point out that both (11) and (2) have been obtained taking into account the hypothesis that the two beams have, locally, the same relative velocity ${ }^{6}$ (in absolute value) in the reference frame defined by the congruence $\Gamma$. In particular, if we refer to a rotating frame in flat space-time,

\footnotetext{
${ }^{5}$ Greek indices run from 0 to 3 , Latin indices run from 1 to 3 , the signature of the space-time metric is $(-1,1,1,1)$.

${ }^{6}$ Generally speaking, we deal with wave packets, hence the velocities we refer to, here and henceforth, are the group velocities of these wave packets (see [2, Sec. 3).
} 
this hypothesis coincides with the condition of "equal velocities in opposite directions" that we imposed in order to obtain the Sagnac effect by using a direct approach [22].

In the following, we calculate the Sagnac effect in some curved spacetimes of physical interest (weak field space-time, Kerr space-time, Gödel's universe, Schwarzschild space-time) by applying the formalism described above. The calculations are performed in full details for the case of the spacetime around a weakly gravitating and rotating source, to give an example.

Before going on, let us point out that, when dealing with weak field, Schwarzschild and Kerr space-times, we consider beams counter propagating around the source of gravitational field, in its equatorial plane, along circular space trajectories defined by $r=R=$ constant. In the case of Gödel's universe, there is not a localized source, but matter is everywhere. Nonetheless, because of the spatial homogeneity of Gödel solution we may consider, again, without loss of generality, beams propagating along circular trajectories $r=R=$ constant in the equatorial plane defined by our choice of coordinates.

\subsection{The weak field around a rotating mass}

The space-time around a weakly gravitating object of mass $M$ and angular momentum $\boldsymbol{J}$ is given ${ }^{7}$ by $^{8}$

$$
\begin{aligned}
d s^{2}= & -\left(1-\frac{2 M}{r^{\prime}}\right) d t^{2}+\left(1+\frac{2 M}{r^{\prime}}\right)\left[d r^{\prime 2}+r^{\prime 2}\left(d \vartheta^{\prime 2}+\sin ^{2} \vartheta^{\prime} d \varphi^{\prime 2}\right)\right]+ \\
& -\frac{4 J}{r^{\prime}} \sin ^{2} \vartheta^{\prime} d \varphi^{\prime} d t^{\prime}
\end{aligned}
$$

where the spherical coordinates $\left(t^{\prime}, r^{\prime}, \vartheta^{\prime}, \varphi^{\prime}\right)$ (adapted to a congruence of a asymptotically inertial observers) have been arranged in such a way that the angular momentum is orthogonal to the equatorial plane.

The last term in (44) is responsible for the rotation of the local inertial frames, the so called Lense-Thirring effect (see [29]).

If we apply the transformation (3) to the metric (4), after setting $\vartheta=\frac{\pi}{2}$, we obtain

$$
d s^{2}=-\left[1-\frac{2 M}{r}-\left(1+\frac{2 M}{r}\right) \Omega^{2} r^{2}+\frac{4 J \Omega}{r}\right] d t^{2}+
$$

\footnotetext{
${ }^{7}$ See for instance 30], Sec. 18.1, and also 10].

${ }^{8}$ Here and henceforth, we use geometric units such that $G=c=1$. Nevertheless, we keep $c$ and $G$ in the final results, for the sake of clarity.
} 


$$
+\left(1+\frac{2 M}{r}\right)\left(d r^{2}+r^{2} d \varphi^{2}\right)+2\left[\left(1+\frac{2 M}{r}\right) \Omega r^{2}-\frac{2 J}{r}\right] d \varphi d t
$$

On evaluating the components of the vector field $\gamma(x)$, along the trajectories $r=R=$ constant, we have

$$
\begin{aligned}
\gamma^{0} & =\gamma_{J} \\
\gamma_{0} & =-\left(\gamma_{J}\right)^{-1} \\
\gamma_{\varphi} & =\left[\left(1+\frac{2 M}{R}\right) \Omega R^{2}-\frac{2 J}{R}\right] \gamma_{J}
\end{aligned}
$$

where

$$
\gamma_{J} \doteq\left[1-\frac{2 M}{R}-\left(1+\frac{2 M}{R}\right) \Omega^{2} R^{2}+\frac{4 J \Omega}{R}\right]^{-1 / 2},
$$

and the corresponding component of the gravito-magnetic vector potential is

$$
\widetilde{A}_{\varphi}^{G}=-\left[\left(1+\frac{2 M}{R}\right) \Omega R^{2}-\frac{2 J}{R}\right] \gamma_{J}^{2}
$$

On using physical units, the phase shift (11) turns out to be

$$
\Delta \Phi=\frac{4 \pi m}{\hbar}\left[\left(1+\frac{2 G M}{c^{2} R}\right) \Omega R^{2}-\frac{2 G J}{c^{2} R}\right] \gamma_{J}
$$

and the time delay (2) is

$$
\Delta T=\frac{4 \pi}{c^{2}}\left[\left(1+\frac{2 G M}{c^{2} R}\right) \Omega R^{2}-\frac{2 G J}{c^{2} R}\right] \gamma_{J}
$$

or, by explicitly writing $\gamma_{J}$

$$
\Delta T=\frac{4 \pi}{c^{2}} \frac{\left[\left(1+\frac{2 G M}{c^{2} R}\right) \Omega R^{2}-\frac{2 G J}{c^{2} R}\right]}{\left[1-\frac{2 G M}{c^{2} R}-\left(1+\frac{2 G M}{c^{2} R}\right) \frac{\Omega^{2} R^{2}}{c^{2}}+\frac{4 G J \Omega}{c^{4} R}\right]^{1 / 2}} .
$$

In (9) and (10) we can distinguish two contributions: the first one is proportional to the angular velocity $\Omega$ of the observer, and the other one depends on the absolute value of the angular momentum of the source $J$.

We see that even when $\Omega=0$ a time difference appears: this is due to the rotation of the source of the gravitational field. In other words

$$
\Delta T_{J} \doteq \frac{4 \pi}{c^{2}} \frac{\left(-\frac{2 G J}{c^{2} R}\right)}{\left(1-\frac{2 G M}{c^{2} R}\right)^{1 / 2}}
$$


is what a non rotating observer would obtain when measuring the propagation time for a complete round trip of the two beams, moving in opposite direction along circular orbits $r=R=$ constant. This time difference corresponds to the so called gravito-magnetic time delay, which has been obtained by Stodolski [17], Cohen-Mashhoon [23] in weak field approximation, and by Tartaglia 24, by a first order approximation of the time delay in Kerr space-time.

By inspection of the time delay (11) we recognize that there is a critical angular velocity $\bar{\Omega}$ such that $\Delta T=0$ :

$$
\bar{\Omega}=\frac{1}{R^{2}} \frac{\frac{2 G J}{c^{2} R}}{1+\frac{2 G M}{c^{2} R}} .
$$

$\bar{\Omega}$ corresponds to the angular velocity of the "Locally Non Rotating Observers" 9

$$
\Omega_{L N R O} \doteq-\frac{g_{t \varphi}}{g_{\varphi \varphi}}
$$

of the weak field described by the metric (4).

These observers measure no time delay. A similar angular velocity can be obtained also in Kerr space-time [24].

\subsection{Kerr space-time}

Kerr solution [31] of Einstein equations describes the space-time around a rotating black-hole or, more generally speaking, around a rotating singularity. The classical form of this solution is given in Boyer-Lindquist coordinates 32] $\left(t^{\prime}, r^{\prime}, \vartheta^{\prime}, \varphi^{\prime}\right)$, which are adapted to a congruence of asymptotically inertial observers

$$
\begin{aligned}
d s^{2}= & -\left(1-\frac{2 M r^{\prime}}{\rho^{2}}\right) d t^{\prime 2}+\frac{\rho^{2}}{\Delta} d r^{\prime 2}+\rho^{2} d \vartheta^{\prime 2}+ \\
& +\left(r^{\prime 2}+a^{2}+\frac{2 M r^{\prime} a^{2} \sin ^{2} \vartheta^{\prime}}{\rho^{2}}\right) \sin ^{2} \vartheta^{\prime} d \varphi^{\prime 2}-\frac{4 M r^{\prime} a \sin ^{2} \vartheta^{\prime}}{\rho^{2}} d \varphi^{\prime} d t^{\prime}
\end{aligned}
$$

where $\rho^{2}=r^{\prime 2}+a^{2} \cos ^{2} \vartheta^{\prime}, \Delta=r^{\prime 2}-2 M r^{\prime}+a^{2}, a=J / M c, J$ is the absolute value of the angular momentum, and the coordinates are arranged in such a way that the angular momentum is perpendicular to the equatorial plane.

\footnotetext{
${ }^{9}$ See, for instance, [30], page 895 .
} 
On applying the prescription described above, the phase shift turns out to be

$$
\Delta \Phi=\frac{4 \pi m c}{\hbar} \frac{\left[-\frac{2 G M a}{c^{2} R}+\left(R^{2}+a^{2}+\frac{2 G M a^{2}}{c^{2} R}\right) \frac{\Omega}{c}\right]}{\left[1-\frac{2 G M}{c^{2} R}+\frac{4 G M a}{c^{2} R} \frac{\Omega}{c}-\left(R^{2}+a^{2}+\frac{2 G M a^{2}}{c^{2} R}\right) \frac{\Omega^{2}}{c^{2}}\right]^{1 / 2}},
$$

and the corresponding time delay is

$$
\Delta T=\frac{4 \pi}{c} \frac{\left[-\frac{2 G M a}{c^{2} R}+\left(R^{2}+a^{2}+\frac{2 G M a^{2}}{c^{2} R}\right) \frac{\Omega}{c}\right]}{\left[1-\frac{2 G M}{c^{2} R}+\frac{4 G M a}{c^{2} R} \frac{\Omega}{c}-\left(R^{2}+a^{2}+\frac{2 G M a^{2}}{c^{2} R}\right) \frac{\Omega^{2}}{c^{2}}\right]^{1 / 2}} .
$$

The time delay (17) is in agreement with the results obtained by Tartaglia 24], who studied in full details the GR corrections to the Sagnac effect in Kerr space-time.

\subsection{Gödel's universe}

Gödel's solution of Einstein's field equation describes, roughly speaking, a rotating universe 33. This solution, indeed, is not satisfactory on the observational viewpoint, since it does not agree with the expansion of the universe and with the cosmological observations of the cosmic background radiation [34]. Another peculiarity of Gödel's solutions is that it admits closed time-like curves [35]. Despite these pathologies, recently there has been some interest about this exact solution of Einstein equations 25. As for us, since this solution describes a rotating universe, it proves useful for studying the corresponding gravito-magnetic features and, among them, the Sagnac effect.

The metric of the Gödel's solution can be written in the form [25]

$$
d s^{2}=-d t^{\prime 2}+\frac{d r^{\prime 2}}{1+\left(\frac{r^{\prime}}{2 a}\right)^{2}}+r^{\prime 2}\left[1-\left(\frac{r^{\prime}}{2 a}\right)^{2}\right] d \varphi^{\prime 2}+d z^{\prime 2}-2 r^{\prime 2} \frac{1}{\sqrt{2} a} d t^{\prime} d \varphi^{\prime}
$$

where cylindrical coordinates $\left(t^{\prime}, r^{\prime}, \varphi^{\prime}, z^{\prime}\right)$ have been used and $a>0$ is a constant having the unit of a length. In our analysis, we confine ourselves to the cylinder $r^{\prime}<2 a$, in order to avoid problems with the chrono-geometric structure of the Gödel's metric.

Then, following the approach described before, the phase shift becomes

$$
\Delta \Phi=\frac{4 \pi m}{\hbar} \frac{\left\{\Omega_{G}+\left[1-\left(\frac{R}{2 a}\right)^{2}\right] \Omega\right\} R^{2}}{\left\{1-\left[1-\left(\frac{R}{2 a}\right)^{2}\right] \frac{R^{2} \Omega^{2}}{c^{2}}-2 \frac{R^{2} \Omega \Omega_{G}}{c^{2}}\right\}^{1 / 2}},
$$


where

$$
\Omega_{G} \doteq-\frac{c}{\sqrt{2} a} .
$$

The corresponding time delay turns out to be

$$
\Delta T=\frac{4 \pi}{c^{2}} \frac{\left\{\Omega_{G}+\left[1-\left(\frac{R}{2 a}\right)^{2}\right] \Omega\right\} R^{2}}{\left\{1-\left[1-\left(\frac{R}{2 a}\right)^{2}\right] \frac{R^{2} \Omega^{2}}{c^{2}}-2 \frac{R^{2} \Omega \Omega_{G}}{c^{2}}\right\}^{1 / 2}} .
$$

The time delay (21) is in agreement with the results given by Kajari et al 25. ${ }^{10}$

Also in this case, we may obtain the velocity of rotation of the observers that measure no time delay, i.e. the "Locally Non Rotating Observers". In agreement with (14), from (21), we get

$$
\bar{\Omega}=-\frac{\Omega_{G}}{1-\left(\frac{R}{2 a}\right)^{2}} .
$$

The previous results, in practice, refer to the physical situation in which the observer (or the detector) uniformly rotates relative to the cosmological fluid, with angular velocity $\Omega$. So, if we want to consider an observer at rest with respect to the cosmological fluid of the Gödel universe, it is sufficient to set $\Omega=0$ in the previous formulae. Consequently the phase shift turns out to be

$$
\Delta \Phi=\frac{4 \pi m}{\hbar} \Omega_{G} R^{2}
$$

and the corresponding time delay is

$$
\Delta T=\frac{4 \pi}{c^{2}} \Omega_{G} R^{2}
$$

Also the time delay (24) is in agreement with the results by Kajari et al. 25.

In these calculations we have formally introduced the quantity $\Omega_{G}$, whose dimensions are those of an angular velocity. It is possible to show that $\Omega_{G}$ is the only non null components of the vortex vector (see [2]) of the congruence of observers at rest with respect to the cosmological fluid. The vortex vector gives the (local) rate of rotation ${ }^{11}$ of the set of the neighbouring particles of the congruence relative to the (local) compass of inertia. In other words,

\footnotetext{
${ }^{10}$ Notice that their definition of $\Omega_{G}$ has a different sign with respect to ours.

${ }^{11}$ With respect to the proper time at any particle of the reference fluid.
} 
the (constant) angular velocity $\Omega_{G}$ expresses the rotation of the particles of the reference fluid with respect to local gyroscopes. This means that, in Gödel's universe, matter rotates with constant angular velocity in the local inertial frames of its comoving observers. The time delay (24) is an effect of this rotation.

\subsection{Schwarzschild space-time}

The standard form of the classical Schwarzschild solution, describing the vacuum space-time around a spherically symmetric mass distribution ${ }^{12}$ is

$$
d s^{2}=-\left(1-\frac{2 M}{r^{\prime}}\right) d t^{\prime 2}+\left(1-\frac{2 M}{r^{\prime}}\right)^{-1} d r^{2}+r^{2}\left(d \vartheta^{\prime 2}+\sin ^{2} \theta^{\prime} d \varphi^{\prime 2}\right),
$$

where $M$ is the mass of the source, and the coordinate $\left(t^{\prime}, r^{\prime}, \vartheta^{\prime}, \varphi^{\prime}\right)$ are adapted to a congruence of asymptotically inertial observers. In this case, our approach to the study of the Sagnac effect leads to the phase shift

$$
\Delta \Phi=\frac{4 \pi m}{\hbar} \frac{\Omega R^{2}}{\left(1-\frac{2 G M}{R c^{2}}-\frac{\Omega^{2} R^{2}}{c^{2}}\right)^{1 / 2}},
$$

and the corresponding time delay turns out to be

$$
\Delta T=\frac{4 \pi}{c^{2}} \frac{\Omega R^{2}}{\left(1-\frac{2 G M}{R c^{2}}-\frac{\Omega^{2} R^{2}}{c^{2}}\right)^{1 / 2}} .
$$

We see that if $\Omega=0$, i.e. if we measure the propagation time in a non rotating frame, no Sagnac effect arises. In other words, the propagation is symmetrical in both directions.

\section{Conclusions}

In the context of natural splitting, the relative formulation of dynamics can be expressed in terms of GEM fields; this analogy, which holds in full theory, leads to the formulation of the GM Aharonov-Bohm effect. We have exploited this fact to give a derivation of the Sagnac effect based on this formal analogy, in stationary and axially symmetric geometries. In doing so, we have showed that the Sagnac effect can be interpreted as a GM Aharonov-Bohm effect in both flat and curved space-time.

\footnotetext{
${ }^{12}$ See, for instance, [30], Sec.23.6.
} 
In particular, we have studied the GR corrections to the Sagnac effect in the space-time around a weakly gravitating and rotating source, in Kerr space-time, in Gödel universe and in Schwarzschild space-time.

Our results are in agreement with those available in the literature and generalize some old approaches, where the analogy with the Aharonov-Bohm effect was limited to a first order approximation.

The Sagnac effect has a "universal" character: in other words, the Sagnac time-delay is the same, independently of the physical nature and velocities of the interfering beams, provided that the latter are locally the same, in absolute value, as seen in the rotating frame, as we explained in full details, by using a direct approach, in 22]. In our formalism this universality is expressed by eq. (2), where the mass (or the energy) of the particles of the beams does not appear, and the time delay is explained as an effect of the geometrical background in which the beams propagate: in this fact we recognize the close analogy with the Aharonov-Bohm effect, which has a purely geometrical interpretation 21. Hence, the geometrical approach clearly points out the universal character of the Sagnac effect.

Acknowledgments: The author would like to thank two anonymous referees for their suggestions which contributed to improve the paper.

\section{References}

[1] Rizzi G., Ruggiero M.L., Gen. Rel. Grav. 35, 1743 (2003), gr-qc/0305046

[2] Rizzi G., Ruggiero M.L., in Relativity in Rotating Frames, eds. Rizzi G. and Ruggiero M.L., in the series "Fundamental Theories of Physics", Kluwer Academic Publishers, Dordrecht (2004), gr-qc/0305084

[3] Cattaneo C., Introduzione alla teoria einsteiniana della gravitazione, Veschi, Roma (1961)

[4] Cattaneo C., Il Nuovo Cimento 10, 318 (1958)

[5] Cattaneo C., Il Nuovo Cimento 11, 733 (1959)

[6] Cattaneo C., Il Nuovo Cimento 13, 237 (1959)

[7] Cattaneo C., Rend. Acc. Lincei 27, 54 (1959) 
[8] Mashhoon B., Gronwald F., Lichtenegger H.I.M., Lect. Notes Phys. 562, 83 (2001), gr-qc/9912027

[9] Jantzen R.T., Carini P., Bini D., Annals of Physics 215, 1 (1992), gr-qc/010604

[10] Ruggiero M.L., Tartaglia A., Il Nuovo Cimento B 117, 743 (2002), gr-qc/0207065

[11] Tartaglia A., in Proceedings of the 16th SIGRAV Conference on General Relativity and Gravitational Physics, eds. Esposito G., Lambiase G., Marmo G., Scarpetta G., Vilasi G., AIP Conference Proceedings, Melville, New York (2005)

[12] Aharonov Y., Carmi G., Found. Phys. 3, 493 (1973)

[13] Sakurai J.J., Phys. Rev. D 21, 2993 (1980)

[14] Semon M. D., Found. Phys. 12, 49 (1982)

[15] Papini G., Nuovo Cimento B 52, 136 (1967)

[16] Wisnivesky D., Aharonov Y., Ann. Phys. 45, 479 (1967)

[17] Stodolsky L., Gen. Rel. Grav. 11, 391 (1979)

[18] Anandan J., Phys. Rev. D. 15, 1448 (1977)

[19] Aharonov Y. and Bohm D., Phys. Rev. 115, 485 (1959)

[20] Anandan J., Phys. Rev. D 24, 338 (1981)

[21] Tourrenc P., Phys. Rev. D 16, 3421 (1977)

[22] Rizzi G., Ruggiero M.L., Gen. Rel. Grav. 35, 2129 (2003), gr-qc/0306128

[23] Cohen J.M., Mashhoon B., Phys. Lett. A 181, 353 (1993)

[24] Tartaglia A., Phys. Rev. D 58, 064009 (1998)

[25] Kajari E. et al., Gen. Rel. Grav. 36, 2289 (2004), gr-qc/0404032

[26] Hehl F.W. and Ni W.T., Phys. Rev. D 42, 2045 (1990)

[27] Mashhoon B., Phys. Rev. Lett. 61, 2639 (1988) 
[28] Papini G., in Relativity in Rotating Frames, ed. Rizzi G. and Ruggiero M.L., in the series "Fundamental Theories of Physics", Kluwer Academic Publishers, Dordrecht (2004)

[29] Lense J., Thirring H., Phys. Z. 19, 156 (1918); the English translation can be found in Mashhoon B., Hehl F.W., Theiss D.S., Gen. Rel. Grav. 16, 711 (1984)

[30] Misner C.W., Thorne K.S., Wheeler J.A., Gravitation, Freeman, S.Francisco (1973)

[31] Kerr R.P. , Phys. Rev. Lett 11, 237 (1963)

[32] Boyer R.H., Lindquist R.W., J. Math. Phys 8, 265 (1967)

[33] Gödel K., Rev. Mod. Phys. 21, 447 (1949)

[34] Bunn E.F., Ferreira P.G., Silk J., Phys. Rev. Lett. 77, 2883 (1996)

[35] Hawking S.W., Ellis G.F.R., The large scale structure of space-time, Cambridge University Press, Cambridge (1973) 